\title{
Distinguishing between enamel fluorosis and other enamel defects in permanent teeth of children
}

Aira Sabokseir, Ali Golkari, Aubrey Sheiham

Background: The inconsistent prevalence of fluorosis for a given level of fluoride in drinking water suggests developmental defects of enamel (DDEs) other than fluorosis were being misdiagnosed as fluorosis. The imprecise definition and subjective perception of fluorosis indices could result in misdiagnosis of dental fluorosis. This study was conducted to distinguish genuine fluorosis from fluorosis-resembling defects that could have adverse health-related events as a cause using Early Childhood Events Life-grid method (ECEL).

Methods: A study was conducted on 400 9-year-old children from areas with high, optimal and low levels of fluoride in the drinking water of Fars province, Iran. Fluorosis cases were diagnosed on the standardized one view photographs of the anterior teeth using Dean's and TF (Thylstrup and Fejerskov) Indices by calibrated dentists. Agreements between examiners were tested. Early childhood health-related data collected retrospectively by ECEL method were matched with the position of enamel defects. Results: Using both Dean and TF indices three out of four dentists diagnosed that $31.3 \%$ (115) children had fluorosis, $58.0 \%, 29.1 \%$, and $10.0 \%$ in high (2.12-2.85ppm), optimal (0.62-1.22ppm), and low $(0.24-0.29 \mathrm{ppm})$ fluoride areas respectively $(\mathrm{p}<0.001)$. After matching health-related events in the $115(31.3 \%)$ of children diagnosed with fluorosis, 31 (8.4\%) of children had fluorosis which could be matched with their adverse health-related events. That suggests that what was diagnosed as fluorosis were non-fluoride related DDEs that resemble fluorosis. Discussion: The frequently used measures of fluorosis appear to overscore fluorosis. Use of ECEL method to consider health related events relevant to DDEs could help to differentiate between genuine fluorosis and fluorosis-resembling defects. 
1 Distinguishing between enamel fluorosis and other enamel defects in permanent 2 teeth of children

$3 \quad$ Aira Sabokseir $^{1}$, Ali Golkari ${ }^{1 *}$, Aubrey Sheiham $^{2}$

41 Department of Dental Public Health, School of Dentistry, Shiraz University of

5 Medical Sciences, Shiraz, Iran,

62 Department of Epidemiology and Public Health, University College London, 7 London, UK.

$9 \quad{ }^{*}$ Corresponding author: Ali Golkari, Shiraz School of Dentistry, Ghomabad,

10 Ghasrodasht St. Shiraz, Iran. Phone: +98 9175607254 Fax: +98 7136270325 e-mail:

11 golkaria@sums.ac.ir 
13

14

15

16

17

\section{Abstract}

Background: The inconsistent prevalence of fluorosis for a given level of fluoride in drinking water suggests developmental defects of enamel (DDEs) other than fluorosis were being misdiagnosed as fluorosis. The imprecise definition and subjective perception of fluorosis indices could result in misdiagnosis of dental fluorosis. This study was conducted to distinguish genuine fluorosis from fluorosis-resembling defects that could have adverse health-related events as a cause using Early Childhood Events Lifegrid method (ECEL).

Methods: A study was conducted on 400 9-year-old children from areas with high, optimal and low levels of fluoride in the drinking water of Fars province, Iran. Fluorosis cases were diagnosed on the standardized one view photographs of the anterior teeth using Dean's and TF (Thylstrup and Fejerskov) Indices by calibrated dentists.

Agreements between examiners were tested. Early childhood health-related data collected retrospectively by ECEL method were matched with the position of enamel defects.

Results: Using both Dean and TF indices three out of four dentists diagnosed that $31.3 \%$ (115) children had fluorosis, $58.0 \%, 29.1 \%$, and $10.0 \%$ in high $(2.12-2.85 \mathrm{ppm})$, optimal $(0.62-1.22 \mathrm{ppm})$, and low $(0.24-0.29 \mathrm{ppm})$ fluoride areas respectively $(\mathrm{p}<0.001)$. After matching health-related events in the 115 (31.3\%) of children diagnosed with fluorosis, 31 (8.4\%) of children had fluorosis which could be matched with their adverse health-related events. That suggests that what was diagnosed as fluorosis were nonfluoride related DDEs that resemble fluorosis.

Discussion: The frequently used measures of fluorosis appear to overscore fluorosis. Use of ECEL method to consider health related events relevant to DDEs could help to differentiate between genuine fluorosis and fluorosis-resembling defects. 
39 Introduction:

40 Despite the extensive use of well documented indices of dental fluorosis (Dean 1942;

41 Fejerskov 1988), there is inconsistency in the reports on the prevalence rates of fluorosis for a given level of fluoride in drinking water. Most probably, this inconsistency in the prevalence of fluorosis occurs due to subjective perception of fluorosis by examiners. Therefore, there is a strong possibility that other Developmental Defects of Enamel (DDE) rather than excess intake of fluoride are being misdiagnosed as fluorosis (Atar \& Körperich 2010).

The two widely used indices of dental fluorosis are Dean's Index (Dean 1934; Dean 1942) and the Thylstrup and Fejerskov Index (TF Index or TFI) (Thylstrup \& Fejerskov 1978; Fejerskov 1988). None of them clearly distinguish between defects caused by fluorosis and caused by other factors. The differences between some of the diagnostic categories are uncertain, vague, or insensitive (a clear example is the "questionable" category in Dean's Index) . In Dean's Index, each individual is given one score, as a score for the whole mouth, according to the two teeth most affected by fluorosis. This index categorizes each tooth as normal, questionable, very mild, mild, moderate, moderately severe, and severe. The classification is based on color and extent of discolored enamel together with added hypoplasia in case tooth belongs to the last two categories.

Thylstrup and Fejerskov reformulated Dean' Index as the TF Index (TFI). They used both clinical and histological appearance of fluorosis and created a single coded index from 0 (normal) to 9 (Thylstrup \& Fejerskov 1978). The TFI was modified and finalized in 1988 (Fejerskov 1988). From 1998, the scoring of facial surface was recommended for TFI. Teeth should be cleaned and dried before examination. Cleaning and drying of teeth make the appearance of fluorotic change more prominent and also the diagnosis of questionable cases easier. Still the difference between some categories of TFI is not clear. 
67 As the main indices for diagnosing dental fluorosis include definitions that are imprecise, 68 it is not surprising that in some studies the reported prevalence of fluorosis was similar despite the levels of fluoride in the drinking water being similar or different. For example, a study in KwaNdebele (Africa) revealed that the prevalence of fluorosis was similar in residents of areas with considerably different levels of fluoride in the water supply (Lewis \& Chikte 1995). A study in Andhra Pradesh (India) of four different areas with different levels of fluoride in drinking water $(<0.7,0.7-1.2,1.3-4.0,>4 \mathrm{ppm})$ reported $100 \%$ dental fluorosis even in areas with optimum level of fluoride (Sudhir et al. 2009). In Hong Kong, where the fluoride concentration of public water supplies was increased from $0.5 \mathrm{ppm}$ to $0.7 \mathrm{ppm}$, and then $1 \mathrm{ppm}$, the prevalence of DDE decreased significantly from $92 \%$, to $55 \%$, and then $35 \%$ (Wong et al. 2006).

DDEs can be localized or generalized. Numerous systemic risk factors cause generalized or "diffuse" DDEs (Small \& Murray 1978; Pindborg 1982; Atar \& Körperich 2010). The generalized DDEs may be of genetic origin (Thesleff 2000; Atar \& Körperich 2010) or caused by malnutrition or diseases that occurred during early childhood (Pindborg 1982; Atar \& Körperich 2010). Fluorosis and some other generalized DDEs are similar in appearance (Small \& Murray 1978; Pindborg 1982). The inconsistent reports on the prevalence of fluorosis suggest that non-fluoride related DDEs were being misdiagnosed as fluorosis. Using teeth as markers of significant health related events and considering these risk factors could help to distinguish between genuine fluorosis and non-fluoride related DDEs that resemble fluorosis.

Genuine fluorosis can best be distinguished from other DDEs by relating a DDE to particular life events, such as a significant health related event. Such events can be reasonably recorded using the life-grid method that helps people remember past events more accurately (Blane 1996). The life-grid method has been widely used and been very successful in obtaining the precise timing of past events in both qualitative and quantitative studies (Berney \& Blane 1997; Holland et al. 2000; Bell 2005). A specially designed life-grid for early childhood containing developmental milestones and shorter 
95 96

97 98

periods of time for the earlier years, is the Early Childhood Events Life-grid method (ECEL) (Golkari 2009).

As there is a need for accurate data of dental fluorosis, the inconsistency of reports on the prevalence of fluorosis suggests that more precise definitions and diagnostic methods are needed for diagnosing dental fluorosis and distinguishing enamel fluorosis from other DDEs. Therefore a study was planned with the objective of distinguishing genuine fluorosis from fluorosis-resembling defects that could have adverse healthrelated events as a cause, instead of just excess fluoride intake.

\section{Materials and methods:}

This cross-sectional study was performed on 400 9-year-old children of Fars province, Iran. The children were randomly selected from areas of Iran with high, optimal and low levels of fluoride in the drinking water. Fluorosis cases were diagnosed using Dean's Index and TF Index (Dean 1942; Fejerskov 1988) by calibrated dentists. To differentiate between genuine fluorosis and fluorosis resembling defects, early childhood data were collected retrospectively by the ECEL method (Golkari 2009).

Ethical permission was obtained from the Ethical Committee of Shiraz University of Medical Sciences (SUMS) and the Educational Head Office of the Fars Province. A written consent form explaining the objectives and the stages of the study were sent to the parents of the selected children. They were included only if the parents agreed to take part.

The level of fluoride in water of each town of the province was obtained from their primary health care trust. Towns were categorized into one of three fluoride categories; high, optimal, and low fluoride. One town was selected randomly from each category. The selected towns were Gerash with high fluoride (2.12- 2.85 ppm) in water, Sepidan 
120 with low fluoride $(0.24-0.29 \mathrm{ppm})$ in water, and Shiraz was the city with an optimal

121 range of fluoride $(0.62-1.22 \mathrm{ppm})$ in water .

122 Sample size was calculated based on the estimated prevalence of fluorosis in Iran

$123(61 \%), d=6.1 \%$ ( $10 \%$ expected prevalence), $\alpha=0.05$. As a result, a sample size of 246

124 was needed if a simple randomized sampling was used in all areas. The selection of

125 children in Gerash and Sepidan (with high and low fluoride levels) was done by simple

126 randomized sampling. However, in Shiraz (chosen as area with optimum fluoride level),

127 the simple randomization was not possible as it was a big city. Therefore, a stratified

128 randomization method had to be adopted. It was done using the four different

129 educational zones of the city. The number of required sample in this area was multiplied

130 by $2(k=2)$ to increase the accuracy in the stratified sampling method that was used. The

131 response rate was suggested to be around $80 \%$. Therefore, at the end, 100 9-year-old

132 schoolchildren of Gerash, 100 from Sepidan and 200 from Shiraz were selected from

133 lists of students obtained from Educational Head Office of each town.

134 Children aged from 9 years, 0 month to 9 years and 11 months who returned the signed 135 consent form were included in the study. Children were excluded if they had lived for more than six months from birth to 5 years of age in other towns (determined during interview with parents). Those who had less than 7 permanent incisor teeth, those with orthodontic brackets, overlapping teeth, and large restorations or severe extrinsic stains on their incisors were also excluded (determined during intra-oral examination).

Intra-oral examinations were carried out to select children conforming to the study criteria. The examinations were performed using a headlight, disposable mirrors and tongue blades with children seated on a chair. Photographs of the dentition of selected children were taken for the diagnosis of dental fluorosis. A one-view photograph was taken of the anterior part of dentition using a digital camera (Nikon D7100, AF-S VR Micro-Nikkor 105mm f/ $2.8 \mathrm{G}$ IF-ED) based on methods described by Wong et al. (2005). Children were asked to close their incisor teeth edge to edge. Cheeks and lips were retracted so that all anterior teeth and some parts of upper and lower gums were visible. The camera was adjusted to 15 degrees above the perpendicular to the central 
149 incisors' plane to minimize specula reflection and burn outs (Ellwood et al. 1996).

150 Immediately after taking each photograph, it was assessed to confirm its quality, and was repeated if necessary.

152 During the fluorosis assessment phase, first, the photographs were randomly ordered to 153 prevent bias induced by the assessors' foreknowledge of the fluoride in the area.

154 Photographs were then assessed by eight calibrated dentists who were blind to the

155

156

157

158

159

160

161

162

163

164

165

166

167

168

169

170

171

172

173

174

175

176

177 clinical condition and town of residence of the subjects. Four calibrated dentists assessed the photographs based on Dean's Index, and another four used the TF Index (Dean 1942; Fejerskov 1988). All dentists observed the photographs on one computer with identical settings. The diagnosis of fluorosis was confirmed only if three out of four dentists of each group agreed.

The objective of the next stage of study was to obtain data on early childhood adverse events. The parents of all children participating in the study were invited to school for interview. Name and date of birth of each child were double checked with their parents. Parents were interviewed using the ECEL method (Golkari 2009). Information regarding gestational age (preterm, term, delayed), birth weight, number of births, type of delivery (natural, caesarean or facilitated delivery), trauma to baby during birth, and newborn vitality score was obtained. Questions were asked relating to personal life line, residential status, occupation of parents/guardians, and child activity line which could help parents to remember adverse health-related events. Afterwards, any illnesses the child had suffered were recorded. For each illness the parent was asked about the name or description of illness, age at which the illness started, duration, perception of severity (mild, moderate, or severe), if went to doctor, medication if used, and hospitalization. Information regarding hospitalizations (age, duration, reason, type of anesthesia if used, and name of hospital), and falls and accidents (age, cause, trauma to face or teeth, hospitalization, and breathing status right after accident as an indicator of severity well known by parents) were also obtained.

The timing of childhood adverse health-related conditions was matched with the timing of formation and calcification of each part of permanent incisors (Golkari 2009). If no 
178 adverse life condition could be matched to the position of a defect diagnosed as

179 fluorosis, the case was considered as genuine fluorosis. However, if a health-related 180 adverse condition could be matched to an enamel defect diagnosed as fluorosis, the 181 case was considered as fluorosis-resembling defect. Using this method, diagnosed

182 fluorosis defects were divided into genuine fluorosis and fluorosis resembling defects.

SPSS software (version 22) was used for data analysis. Agreements between examiners who assessed fluorosis using Dean's Index and TF Index were tested using Kappa-coefficient. McNemar, and Pearson correlation tests were also used to compare the results reported by each pair of dentists. The prevalence of fluorosis in the three selected areas was compared, before and after adjustments for sex, by chi-square test and logistic regression. Childhood adverse health-related conditions were matched to enamel defects diagnosed as fluorosis one by one.

\section{Results:}

The number of 9-year-old children included in the study was 376; 171 (46\%) girls and 196 (53 \%) boys. The number of included children from Gerash (high F), Shiraz (optimal F) and Sepidan (low F) were 88, 189, and 90 respectively .

Using both Dean and TF indices, three out of four dentists diagnosed that 31.3\% (115) of children had fluorosis. The percentage of fluorosis cases in areas with high, optimal, and low range of fluoride in water was $58.0 \%, 29.1 \%$, and $10.0 \%$ respectively $(p<0.001)$. Logistic regression showed that there was a positive relationship between fluorosis and fluoride in the drinking water $(p<0.001)$. There was no relationship between fluorosis and children's sex $(p=0.228)$.

There were significant differences among dentists who scored photographs using Dean's and TF indices. Among the four dentists who assessed photographs according to Dean's Index, the difference in the number of cases diagnosed as fluorosis was statistically different between each two dentists $(p<0.001)$. There was only a slight 
205 (kappa was between 0 and 0.2) or fair (kappa was between 0.2 and 0.4 ) agreement 206 between them (Table 1). Similar results of agreement were observed among the four

207

208

209

210

211

212

213

214

215

216

217

218

219

220

221

222

223

224

225

226

227

228

229

230

dentists who scored children using the TF Index. Although there was not a high agreement among dentists, a positive correlation was observed $(p<0.001)$ (Table 2).

Adverse past childhood health-related events were found in 311 (84.7\%) of children using the ECEL method. Fluorosis defects were found in 115 (31.3\%) of children. The timing of adverse health-related events could be matched with the position of what was diagnosed as fluorosis in $31(8.4 \%)$ of children. These were regarded as fluorosis resembling defects. Therefore, it was concluded that $26.9 \%$ of what was first diagnosed as fluorosis was in fact fluorosis resembling defects.

The overall percentage of genuine fluorosis was $22.9 \%: 47.7 \%, 20.6 \%$, and $3.3 \%$ in areas with high, optimal, and low fluoride areas respectively $(p<0.001)$. The percentage of fluorosis resembling defects in areas with high, optimal, and low range of fluoride in water was $10.2 \%, 8.5 \%, 6.7 \%$ respectively. The difference in percentage of fluorosis resembling defects among the three areas was not statistically significant (Table 3).

\section{Discussion:}

Fluoride is one of the most successful measures for prevention of dental caries in public health (Petersen \& Lennon 2004). However, there has always been controversy about using fluoride because of fluorosis (Sapolsky 1968; Null \& Feldman 2003; Ananian et al. 2005). Reports of a high prevalence of fluorosis in communities have led to objections to fluoride. Therefore, there is a need for a precise way to diagnose dental fluorosis. Many local and systemic risk factors cause DDEs. Some non-fluoride related DDEs are similar to enamel fluorosis and should be differentiated from genuine fluorosis. No standard method has been established to differentiate them from one another. The main 
231 objective of this study was therefore, to try a method to distinguish fluorosis from other

232 kinds of DDEs that look like fluorosis but are caused by adverse health-related events, 233 not by excess fluoride intake.

234

235

236

237

238

239

240

241

242

243

244

245

246

247

248

249

250

251

252

253

254

255

256

A systematic review reported that in Iran with an average concentration of fluoride in water of $0.43 \pm 0.17 \mathrm{ppm}$, the prevalence of fluorosis was $61 \%$ (Azami-Aghdash et al. 2013). Based on the level of fluoride, the reported prevalence of fluorosis was high and questionable. The inconsistency of the prevalence of fluorosis with the level of fluoride in water reported in that study and in many other different parts of the world (Lo \& Bagramian 1996; Sudhir et al. 2009; Arif et al. 2013) suggests that dental fluorosis was misdiagnosed.

The overall prevalence of diagnosed dental fluorosis in the current study was $31.3 \%$. However, by using the ECEL method and considering health-related events, the prevalence of genuine dental fluorosis was $22.6 \%$ as there were $8.4 \%$ with fluorosis resembling defects. That illustrates that fluorosis could be ruled out as the main cause of about $27 \%$ of what was incorrectly diagnosed as fluorosis.

The current study also showed significant differences among the dentists who scored photographs to diagnose fluorosis according to Dean's and TF Indices $(p<0.001)$. This finding indicates that both Dean and TF Indices are too subjective and therefore not precise ways to diagnose dental fluorosis.

These two Indices could lead to misdiagnosis of fluorosis resembling defects as genuine fluorosis. Tavener et al also concluded that interpretation of criteria could be different among examiners and stressed the necessity for standard methods to measure dental fluorosis (Tavener et al. 2007). Some studies have shown good to excellent agreement among examiners (Kumar et al. 2000). However, comparing the methods, the lack of bias was an advantage of the current study, as photographs were assessed instead of clinical examinations. 
257 This study indicates that by considering adverse health-related events, it is possible to 258 distinguish genuine fluorosis from fluorosis-resembling defects. When the timing of an 259 adverse condition matches the timing of development of the part of enamel defect, the

260

261

262

263

264

265

266

267

268

269

270

271

272

273

274

275

276

277

278

279

280

281

282

283

284 adverse event could be the cause of the defect, not fluoride. If no adverse condition could be matched to a defect, excess fluoride could, with caution, be considered as the cause. In case of generalized defects, fluoride, genetic, or severe underlying systematic disease, either individually or as a combination could be the cause.

One limitation of this study is that even if an adverse health-related event could be exactly matched to a fluoride-resembling defect in terms of time and place, it could not be definitely considered that the adverse event was the cause of the defect and fluoride was not the cause. On the other hand, if an adverse condition could not be matched to a supposedly fluorosis defect, one could not be sure that fluoride was the cause. In fact, the definite diagnosis of fluorosis is not possible by this method and the only exact way to diagnose dental fluorosis would be by microscopic or chemical analysis. However, although the ECEL method cannot prove that fluoride is or is not the cause of defect, it can help in differentiate between genuine fluorosis and fluorosis-resembling defects. In the case of this study, past childhood data obtained by the ECEL method helped to find fluorosis-resembling defects in $10.2 \%, 8.5 \%$, and $6.7 \%$ of subjects from high, optimal, and low fluoride areas respectively.

It was decided from the beginning of the study that children with large restorations on their permanent anterior teeth should be excluded as it was not possible to assess the previous existence of defects on them. This was while the authors were aware of the bias this could make. The large restorations could be due to fluorosis, non-fluoride related DDEs, dental caries, trauma or other reasons. One could rule out dental caries and trauma easily by questioning the parents. However, there was no means of distinguishing between fluorosis and any other types of defects. Fortunately, in this study, only one child was excluded based on having large restorations. This one subject 
285 could not affect the results of this study. However, this should be addressed in future 286 studies.

287

288

289

290

291

292

293

294

295

296

297

298

299

300

301

302

303

304

305

306

307

308

The diagnosis of fluorosis is more complicated than acknowledged. On the other hand, there has always been considerable controversy regarding the use of fluoride for dental caries prevention. One of the main issues, which opponents of fluoridation raise, is fluorosis. Therefore, finding a reliable way for more accurate diagnosis of genuine fluorosis is vital. Existing fluorosis indices could lead to misjudgment about using fluoride. However, the ECEL method to record health-related life events is a promising method to help differentiate between genuine fluorosis and fluorosis-resembling defects.

\section{Conclusion:}

Fluorosis indices, if used alone, could result in misdiagnosis of dental fluorosis and misguide health policymakers in their decision about public health measure related to use of fluoride. Information about adverse health-related conditions linked to DDEs at specific positions on teeth could help to differentiate between genuine fluorosis and fluorosis-resembling defects.

\section{Acknowledgements:}

This manuscript is based on ASa PhD thesis which was registered in Shiraz University of Medical Sciences (\# PhD 122) and was conducted under supervision of AG and advisory of ASh. The authors would like to thank Dr Mehrdad Vossoughi for his statistical advice. 
310

311

312

313

314

315

316

317

318

319

320

321

322

323

324

325

326

327

328

329

330

331

332

333

334

335

336

337

338

339

340

341

342

343

344

345

346

347

348

349

350

351

352

353

354

355

\section{References:}

Ananian A, Solomowitz B, and Dowrich I. 2005. Fluoride: a controversy revisited. The New York State Dental Journal 72:14-18.

Arif M, Husain I, Hussain J, and Kumar S. 2013. Assessment of fluoride level in groundwater and prevalence of dental fluorosis in Didwana block of Nagaur district, Central Rajasthan, India. International Journal of Occupation and Environmental Medicine 4:178-184.

Atar M, and Körperich EJ. 2010. Systemic disorders and their influence on the development of dental hard tissues: a literature review. Journal of Dentistry 38:296-306.

Azami-Aghdash S, Ghojazadeh M, Pournaghi Azar F, Naghavi-Behzad M, Mahmoudi M, and Jamali Z. 2013. Fluoride concentration of drinking waters and prevalence of fluorosis in iran: a systematic review. Journal of dental research, dental clinics, dental prospects 7:1-7. DOI: 10.5681 joddd.2013.001

Bell AJ. 2005. 'Oh yes, I remember it well!'Reflections on using the life-grid in qualitative interviews with couples. Qualitative Sociology Review 1:51-67.

Berney LR, and Blane DB. 1997. Collecting retrospective data: accuracy of recall after 50 years judged against historical records. Social Science and Medicine 45:1519-1525.

Blane DB. 1996. Collecting retrospective data: development of a reliable method and a pilot study of its use. Social Science and Medicine 42:751-757.

Dean HT. 1934. Classification of Mottled Enamel Diagnosis. Journal of the American Dental Association 21:1421-1426.

Dean HT. 1942. the investigation of physiological effects by the epidemiological method. In: Moulton FR, ed. Flourine and dental health. Washington DC: American Association for the Advancement of Science.

Ellwood RP, Cortea DF, and O'Mullane DM. 1996. A photographic study of developmental defects of enamel in Brazilian school children. International Dental Journal 46:69-75.

Fejerskov O. 1988. Dental fluorosis : a handbook for health workers. Copenhagen: Munksgaard.

Golkari A. 2009. Developmental defects of enamel as biomarkers of early childhood life events: Developing methods for their use in life course epidemiology. UCL (University College London).

Holland P, Berney L, Blane D, Smith GD, Gunnell DJ, and Montgomery SM. 2000. Life course accumulation of disadvantage: childhood health and hazard exposure during adulthood. Social Science and Medicine 50:1285-1295.

Kumar JV, Swango PA, Opima PN, and Green EL. 2000. Dean's fluorosis index: an assessment of examiner reliability. Journal of Public Health Dentistry 60:57-59.

Lewis HA, and Chikte UM. 1995. Prevalence and severity of fluorosis in the primary and permanent dentition using the TSIF. Journal of Dental Association of South Africa 50:467-471.

Lo GL, and Bagramian RA. 1996. Prevalence of dental fluorosis in children in Singapore. Community Dentistry and Oral Epidemiology 24:25-27.

Null G, and Feldman M. 2003. The Fluoride Controversy Continues: An Update-Part 1. Townsend Letter for Doctors and Patients:117-121.

Petersen PE, and Lennon MA. 2004. Effective use of fluorides for the prevention of dental caries in the 21st century: the WHO approach. Community Dentistry and Oral Epidemiology 32:319-321.

Pindborg JJ. 1982. Aetiology of developmental enamel defects not related to fluorosis. International Dental Journal 32:123-134.

Sapolsky HM. 1968. Science, voters, and the fluoridation controversy. Science 162:427-433.

Small BW, and Murray JJ. 1978. Enamel opacities: prevalence, classifications and aetiological considerations. Journal of Dentistry 6:33-42. 
356

Sudhir KM, Prashant GM, Subba Reddy VV, Mohandas U, and Chandu GN. 2009. Prevalence and severity of dental fluorosis among 13- to 15-year-old school children of an area known for endemic fluorosis: Nalgonda district of Andhra Pradesh. Journal of Indian Society for Pedodontics and Preventive Dentistry 27:190-196. DOI: 10.4103/0970-4388.57651

Tavener J, Davies RM, and Ellwood RP. 2007. Agreement amongst examiners assessing dental fluorosis from digital photographs using the TF index. Community Dental Health 24:21-25.

Thesleff I. 2000. Genetic basis of tooth development and dental defects. Acta odontologica Scandinavica 58:191-194.

Thylstrup A, and Fejerskov O. 1978. Clinical appearance of dental fluorosis in permanent teeth in relation to histologic changes. Community Dentistry and Oral Epidemiology 6:315-328.

Wong HM, McGrath C, Lo EC, and King NM. 2005. Photographs as a means of assessing developmental defects of enamel. Community Dentistry and Oral Epidemiology 33:438-446. DOI: 10.1111/j.1600-0528.2005.00245.x

Wong HM, McGrath C, Lo EC, and King NM. 2006. Association between developmental defects of enamel and different concentrations of fluoride in the public water supply. Caries Research 40:481-486. DOI: 10.1159/000095646 
374 Table1. The comparison of fluorosis scores according to Dean's Index by four calibrated 375 dentists

\begin{tabular}{|c|c|c|c|c|}
\hline & Test & Examiner 2 & Examiner 3 & Examiner 4 \\
\hline \multirow{3}{*}{ Examiner 1} & Kappa value & 0.16 & 0.16 & 0.07 \\
\hline & McNemar p-value & $<0.001$ & $<0.001$ & $<0.001$ \\
\hline & $\begin{array}{l}\text { Correlation } \\
\text { coefficient }\end{array}$ & 0.528 & 0.454 & 0.458 \\
\hline \multirow{4}{*}{ Examiner 2} & Kappa value & & 0.34 & 0.37 \\
\hline & McNemar p-value & & 0.125 & $<0.001$ \\
\hline & Correlation & & 0.584 & 0.575 \\
\hline & coefficient & & & \\
\hline \multirow{4}{*}{ Examiner 3} & Kappa value & & & 0.29 \\
\hline & McNemar p-value & & & 0.003 \\
\hline & Correlation & & & 0.614 \\
\hline & coefficient & & & \\
\hline
\end{tabular}


378 Table 2.The comparison of fluorosis scores according to TF Index by four calibrated 379 dentists

\begin{tabular}{|c|c|c|c|c|}
\hline & Test & Examiner 2 & Examiner 3 & Examiner 4 \\
\hline \multirow{4}{*}{ Examiner 1} & Kappa value & 0.06 & 0.21 & 0.34 \\
\hline & McNemar p-value & $<0.001$ & $<0.001$ & $<0.001$ \\
\hline & Correlation & 0.381 & 0.465 & 0.498 \\
\hline & coefficient & & & \\
\hline \multirow{4}{*}{ Examiner 2} & Kappa value & & 0.27 & 0.18 \\
\hline & McNemar $p$-value & & $<0.001$ & $<0.001$ \\
\hline & Correlation & & 0.472 & 0.503 \\
\hline & coefficient & & & \\
\hline \multirow{4}{*}{ Examiner 3} & Kappa value & & & 0.36 \\
\hline & McNemar $p$-value & & & $<0.001$ \\
\hline & Correlation & & & 0.539 \\
\hline & coefficient & & & \\
\hline
\end{tabular}

380

381 
382 Table 3: Comparison of all defects diagnosed as fluorosis, genuine fluorosis, and 383 fluorosis-resembling defects among the three areas

\begin{tabular}{|c|c|c|c|c|}
\hline & & $\begin{array}{c}\text { All children } \\
\text { diagnosed } \\
\text { with fluorosis* }\end{array}$ & $\begin{array}{l}\text { Children with } \\
\text { genuine } \\
\text { fluorosis }\end{array}$ & $\begin{array}{c}\text { Children with } \\
\text { fluorosis } \\
\text { resembling defects }\end{array}$ \\
\hline \multirow{5}{*}{$\begin{array}{l}\text { Level of } \\
\text { fluoride in } \\
\text { area }\end{array}$} & High $(N=88)$ & $51(58.0 \%)$ & $42(47.7 \%)$ & $9(10.2 \%)$ \\
\hline & Optimal $(\mathrm{N}=189)$ & $55(29.1 \%)$ & $39(20.6 \%)$ & $16(8.5 \%)$ \\
\hline & Low $(\mathrm{N}=90)$ & $9(10.0 \%)$ & $3(3.3 \%)$ & $6(6.7 \%)$ \\
\hline & $\begin{array}{l}\text { OR optimal/high } \\
(95 \% \mathrm{Cl})\end{array}$ & $\begin{array}{c}0.303^{* *} \\
(0.179-0.514)\end{array}$ & $\begin{array}{c}0.292^{* *} \\
(0.168-0.506)\end{array}$ & $\begin{array}{c}0.797 \\
(0.337-1.886)\end{array}$ \\
\hline & $\begin{array}{l}\text { OR low/high } \\
(95 \% \mathrm{Cl})\end{array}$ & $\begin{array}{c}0.080^{* *} \\
(0.036-0.180)\end{array}$ & $\begin{array}{c}0.037^{* *} \\
(0.011-0.127)\end{array}$ & $\begin{array}{c}0.625 \\
(0.231-1.837)\end{array}$ \\
\hline \multirow{3}{*}{ Sex } & Boys $(N=196)$ & $67(34.2 \%)$ & $52(26.5 \%)$ & $15(7.7 \%)$ \\
\hline & Girls $(N=171)$ & $48(28.1 \%)$ & $32(18.7 \%)$ & $16(9.4 \%)$ \\
\hline & $\begin{array}{l}\text { OR girls/boys } \\
(95 \% \mathrm{Cl})\end{array}$ & $\begin{array}{c}0.744 \\
(0.462-1.201)\end{array}$ & $\begin{array}{c}0.619 \\
(0.362-1.056)\end{array}$ & $\begin{array}{c}1.251 \\
(0.597-2.620)\end{array}$ \\
\hline
\end{tabular}

${ }^{*}$ All children that were diagnosed as having fluorosis by three out of four calibrated 385 dentists using both Dean and TF Indices.

$386^{* *}$ Significant at 0.001 level. 